\title{
Hybrid Treatment of Multiple Aortic Aneurysms by Combined Conventional Surgery and Endovascular Aortic Repair
}

\author{
Yukio Obitsu, MD, Nobusato Koizumi, MD, Yasunori Iida, MD, Kazuhiro Satou, MD, Yoshiko Watanabe, MD, \\ Hisahito Takae, MD, Naozumi Saiki, MD, Satoshi Kawaguchi, MD, and Hiroshi Shigematsu, MD
}

Purpose: We evaluated the operative results of our treatment for multiple aortic aneurysms by means of a hybrid procedure consisting of a combination of conventional surgical maneuvers and simultaneous or sequential endovascular aortic repair (EVAR).

Materials and Methods: From August 1998 to April 2007, a total of 15 patients, 11 men and 4 women, ranging in age from 62 to 78 years, were treated with hybrid procedures for multiple aortic aneurysms. The pathology of these patients were, atherosclerotic aneurysm in 12 patients, atherosclerotic aneurysm associated with chronic dissection in 2 and type III chronic dissection in 1 patient. The distribution of aneurysmal locations were as follows: 5 patients had aneurysms at the arch and descending aorta, 1 had at the arch and thoracoabdominal aorta, 6 at the descending and abdominal aorta. Two patients with chronic dissection had simultaneous abdominal aortic aneurysms. In all except 1 of the 7 patients who had abdominal aortic aneurysm, we performed abdominal aneurysmectomy and EVAR simultaneously. In 7 patients, EVAR was performed sequentially after graft replacement surgery. In 2 patients, EVAR was the initial procedure followed by conventional surgery. The mean interval between first and second stage procedures was approximately 4 months.

Results: One patients died of methicillin resistant Staphylococcus aureus pneumonia 3 months after the simultaneous procedure, all others were discharged and survive. In particular, no major EVAR linked complication was observed in the follow-up period (range 3 to 91 months, mean 46 months).

Conclusion: The hybrid procedure for the treatment of multiple aortic aneurysmal disease is less invasive compared to conventional staged surgery and the outcome in terms of mortality and morbidity in hospital as well as long-term follow-up are satisfactory.

Key words: multiple aortic aneurysms, hybrid procedure, endovascular aortic repair, elephant trunk

\section{INTRODUCTION}

$\mathrm{S}$ imultaneous surgery for the treatment of multiple aortic aneurysms is theoretically desirable, however, the high invasiveness involves a high risk for the patient. As an alternative, staged surgery is undertaken in the majority of cases. To minimize the surgical risk, endoDepartment of Vascular Surgery, Tokyo Medical University, Tokyo, Japan

Received: January 30, 2007 Accepted: August 21, 2007 Address for reprint requests to Yukio Obitsu: Department of Vascular Surgery, Tokyo Medical University, 6-7-1 Nishishinjuku, Shinjuku-ku, Tokyo 160-0023, Japan vascular aortic repair (EVAR) has been aggressively introduced and has recently become one of the routine procedures for the treatment of aortic disease. Although EVAR is less invasive, there are of course certain limitations to in application, including the nature of the pathology, location of the lesion etc. In order to reduce the risk of multiple surgeries, while at the same time trying to enhance the outcome of treatment, we developed a hybrid type of procedure consisting of a combination of conventional routine surgery and EVAR for the treatment of multiple aortic aneurysmal diseases. This report describes our experiences with 15 patients who received treatment by this new modality, their surgical outcome 
and follow-up results of the procedure, in an attempt to work forwards the established of the indications.

\section{SubJECTS AND Materials}

Treatment by EVAR for aortic aneurysm was initially introduced at our institution in April, 1995 and treatment with the hybrid procedure was first attempted in August, 1998. We treated a total of 873 patients with thoracic aortic aneurysms during the period from April, 1995 to April, 2007, including 281 patients receiving conventional surgical procedures, 577 patients receiving EVAR and 15 patients treated by the hybrid procedure i. e., combination of the conventional surgical procedure and EVAR, either simultaneously or sequentially in a staged approach, for multiple aortic aneurismal diseases.

Of these 15 patients, 11 patients were men and 4 were women, ranging in age from 62 to 78 years. Most of these patients had atherosclerotic aneurysms, of whom 2 had atherosclerotic aneurysm associated with chronic dissection, and 1 patient had chronic type III aortic dissection. The distribution and location of aneurysmal lesions were as follows: 5 had aneurysms at the aortic arch and the descending aorta, one had aneurysms at the aortic arch and the thoracoabdominal aorta, 6 had aneurysms at the descending and the abdominal aorta, 2 patients with chronic type III dissection were associated with abdominal aortic aneurysms.

The definitive indications for hybrid procedure have not yet been established, thus the selection of patients was determined on an individual base, mainly according to the anatomical indications of the application of EVAR such as the presence of an intact landing zone with a length of at least $20 \mathrm{~mm}$ and an aortic diameter of less than $40 \mathrm{~mm}$. The application of the hybrid procedure to the patients with multiple aortic aneurysms was approved on an individual basis by the Institutional Review Board of our Tokyo Medical University, and the patients provided written informed consent after extensive explanation of the treatment.

All of the hybrid procedures were undertaken either simultaneously in a one-stage approach or sequentially in a staged approach. In 7 of 8 patients with associated simultaneous abdominal aneurysmectomy and EVAR were performed. In 6 patients, EVAR was performed sequentially after graft replacement surgery. In 2 patients, EVAR was applied as the initial procedure followed later by conventional surgery.

In EVAR, stent grafts was handmade by the surgical staff by connecting modified stainless steel $\mathrm{Z}$ stents using two support wires and covering the stents using a polyester artificial prosthesis. Generally such stents were inserted through the femoral artery using a 20-22 F sheath.

\section{Hybrid Procedures}

In 6 patients who had associated descending aortic lesion (4 patients with atherosclerotic aneurysm and 2 patients with chronic type III aortic dissection) and the abdominal aortic aneurysm, hybrid procedures were undertaken simultaneously. The infrarenal abdominal aneurysms were replaced using a bifurcated artificial prosthesis, and stent grafts were inserted, through either the femoral artery or a previously prepared artificial lateral branch to the main graft, to the designated location in the descending aorta. In 9 patients who were sequentially treated, conventional surgical repair was performed first in 7 patients, followed by EVAR, while EVAR was undertaken first followed by surgical replacement in 2 patients. The surgical procedures in this group consisted of total aortic arch replacement in 6 patients and thoracoabdominal aortic replacement in 3 patients. In one patient, the aortic arch and the thoracoabdominal aorta were replaced in a two-stage operation. The stent grafts in this sequential group were placed in the descending aorta or the supra-celiac portion of thoracoabdominal aorta. In 5 patients in whom the total aortic arch was replaced in the first procedure, elephant trunk procedures were used for distal anastomosis and the trunks of the grafts were used as the proximal landing zone for EVAR (Figs. 1, 2). The interval between the first and second stage procedure ranged from 2 weeks up to 18 months, although the second stage procedure was performed less than one month in 5 patients (Table 1).

\section{RESULTS}

One patient (Case 10) who received simultaneous abdominal aortic aneurysmectomy and EVAR of the descending aorta died of multiple organ failure due to pneumonia caused by methicillin resistant Staphylococcus auerus (hospital mortality rate $6.6 \%$ ). The postoperative courses of the other 14 patients were uneventful, with no notable complication. during follow-up observation ranging from 3 to 91 months (mean 46 months), no anastomotic complication, endoleak or migration-induced increase in aneurysmal diameter was seen at the site of the aortic replacement or stent graft. Although in Case 8, an aortic arch aneurysm developed at 7 years after undergoing simultane- 

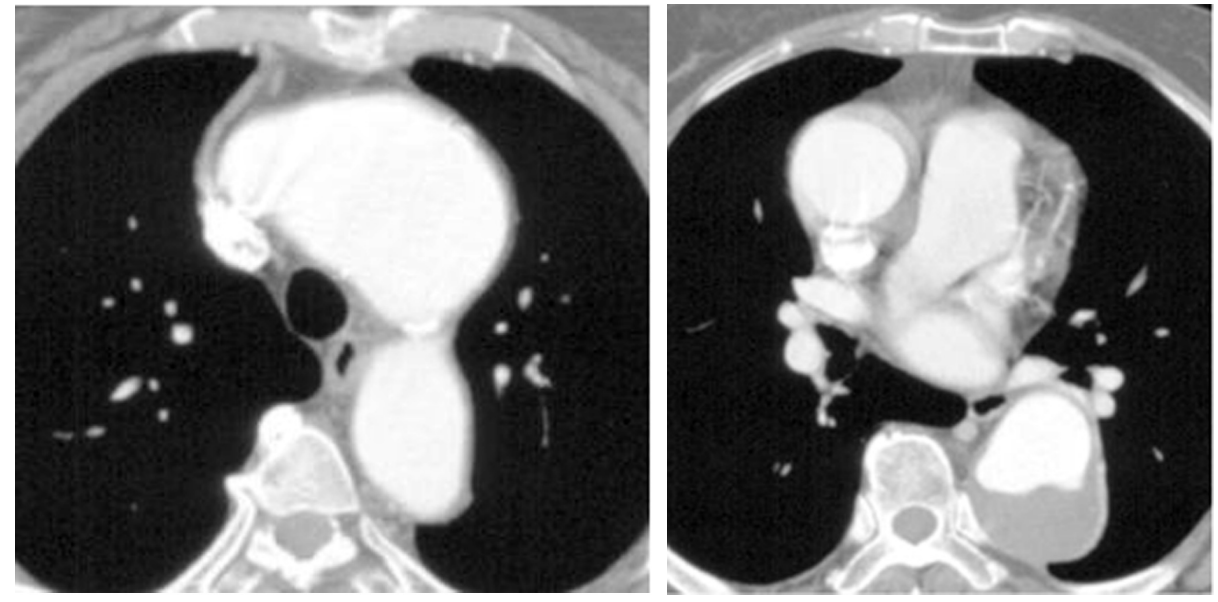

Fig. 1 Preoperative enhanced CT demonstrate aortic arch and descending thoracic aortic aneurysms in a 68 year old female.
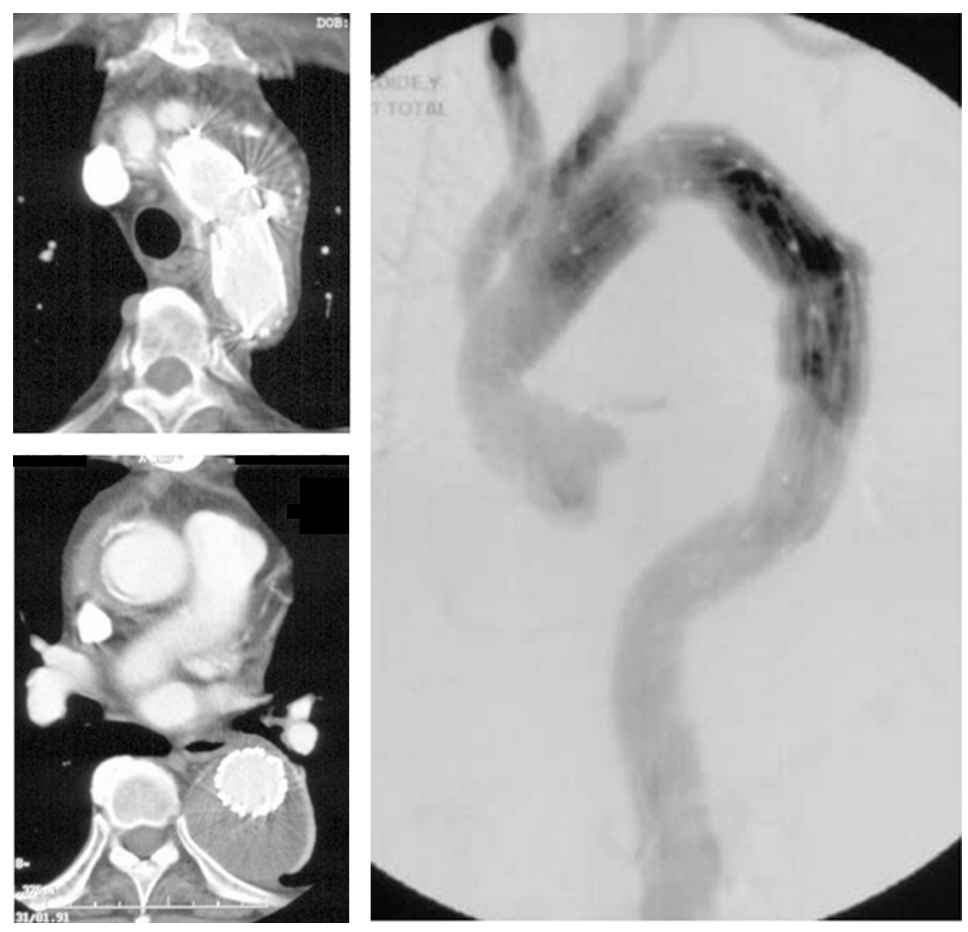

Fig. 2 Postoperative enhanced CT and angiography obtained from the same patient in Fig.1 after combined total aortic arch replacement and secondary endovascular aortic repair for descending thoracic aortic aneurysm.

ous abdominal aortic aneurysmectomy and EVAR, total aortic arch replacement and coronary artery bypass surgery were performed. Two patients died during the followup period of causes unrelated to the aortic lesions.

\section{Discussion}

With the recent increase of atherosclerotic diseases, multiple aortic aneurysms are not rare in the clinical setting and the treatment is usually undertaken either by one-stage or multiple-staged surgery. If aneurysms are located close together, surgery can be performed in simultaneously one stage, however, if aneurysms are located at the aortic arch and abdominal region, they must be treated sequentially by a staged approach, because treating all the aneurysms in one procedure would be exces- 
Table 1 Patient characteristics and clinical outcomes

\begin{tabular}{|c|c|c|c|c|c|}
\hline Case & Age, Sex & Disease & Operation & Clinical coarse & Duration of follow-up \\
\hline 1 & $71, \mathrm{M}$ & TAAarch, TAAdesc & TAR $\rightarrow$ EVG (7M) & good & $17 \mathrm{M}$ \\
\hline 2 & $64, \mathrm{M}$ & TAAarch, TAAdesc & TAR $\rightarrow$ EVG (17M) & good & $50 \mathrm{M}$ \\
\hline 3 & $68, \mathrm{~F}$ & TAAarch, TAAdesc & TAR $\rightarrow$ EVG $(3 \mathrm{~W})$ & good & $25 \mathrm{M}$ \\
\hline 4 & $68, \mathrm{~F}$ & TAAarch, TAAdesc & $\mathrm{TAR} \rightarrow \mathrm{EVG}(3 \mathrm{~W})$ & good & $20 \mathrm{M}$ \\
\hline 5 & $66, \mathrm{~F}$ & TAAarch, TAAA & TAR $\rightarrow$ TAAR $(3 \mathrm{M}) \rightarrow$ EVG $(18 \mathrm{M})$ & good & $52 \mathrm{M}$ \\
\hline 6 & $68, \mathrm{M}$ & TAAarch, TAAdesc & $\mathrm{EVG} \rightarrow \mathrm{TAR}(2 \mathrm{~W})$ & good & $45 \mathrm{M}$ \\
\hline 7 & $69, \mathrm{~F}$ & TAAdesc, TAAA, AAA & $\mathrm{EVG} \rightarrow \mathrm{TAAR}, \mathrm{AAR}(4 \mathrm{~W})$ & good & $11 \mathrm{M}$ \\
\hline 8 & $63, \mathrm{M}$ & TAAdesc, AAA & $\mathrm{AAR}+\mathrm{EVG} \rightarrow \mathrm{TAR}(86 \mathrm{M})$ & good & $89 \mathrm{M}$ \\
\hline 9 & $60, \mathrm{M}$ & TAAdesc, AAA & $\mathrm{AAR}+\mathrm{EVG}$ & good & $66 \mathrm{M}$ \\
\hline 10 & $72, \mathrm{M}$ & TAAdesc, AAA & $\mathrm{AAR}+\mathrm{EVG}$ & dead & $3 \mathrm{M}$ \\
\hline 11 & $75, \mathrm{M}$ & TAAdesc, AAA & $\mathrm{AAR}+\mathrm{EVG}$ & good & $68 \mathrm{M}$ \\
\hline 12 & $74, \mathrm{M}$ & DAA, AAA & $\mathrm{AAR}+\mathrm{EVG}$ & good & $70 \mathrm{M}$ \\
\hline 13 & $78, \mathrm{M}$ & DAA, AAA & $\mathrm{AAR}+\mathrm{EVG}$ & good & $91 \mathrm{M}$ \\
\hline 14 & $69, \mathrm{M}$ & TAA, AAA & $\mathrm{AAR}+\mathrm{EVG}$ & good & $8 \mathrm{M}$ \\
\hline 15 & $62, \mathrm{M}$ & DAA & TAAR $\rightarrow$ EVG $(3 \mathrm{~W})$ & good & $71 \mathrm{M}$ \\
\hline
\end{tabular}

TAA: thoracic aortic aneurysm, TAAA: thoracoabdominal aortic aneurysm, AAA: abdominal aortic aneurysm, DAA: dissecting aortic aneurysm, TAR: total aortic arch replacement, TAAR: thoracoabdominal aortic replacement, AAR: abdominal aortic replacement, EVAR: endovascular aortic repair.

sively invasive, casting the operative outcome into doubt. When staged surgery is planned, the therapeutic priority is determined based on factors such as the aneurysmal size, shape, tendency to increase in size, at the same time, and it is also important to consider the risk of rupture before the next surgery. Two patients who had thoracic and abdominal aortic aneurysm died of rupture before the planned second surgery, therefore, we must be aware of the necessity of performing simultaneous surgery with a less invasive modality in patient with critical multiple aneurysms or shorting interval to the second surgery.

With the recent advances in endovascular therapy, EVAR has been increasingly introduced for the treatment of aortic aneurysm and the initial results are favorable. ${ }^{1)}$ According to the current standards for EVAR, an intact landing zone of at least $20 \mathrm{~mm}$ and an aortic diameter less than $40 \mathrm{~mm}$ are required. Therefore in terms of surgical indications, anatomical factors such as the nature of arterial lesion as well as aneurysmal morphology play an important role, and EVAR should not be undertaken if the aneurysm is located near a major aortic branch. Flexible stent grafts for distal aortic arch aneurysms and branched or fenestrated stent grafts for maintaining the blood flow into the branch have been developed and their clinical applications are being evaluated ${ }^{1-3)}$ but these stent grafts have been used only few in institutions. Our hybrid procedure combining the conventional surgical approach and EVAR has been slowly developed to expand the indi- cations for the application of EVAR, while, trying to minimize the invasiveness of surgery for extensive aortic lesions. Previously reported hybrid procedures include: (1) total aortic arch replacement during the first surgery followed later by EVAR for a descending thoracic aorta lesion using the elephant trunk as the landing zone, ${ }^{4}$ (2) EVAR combined with bypass surgery to secure the landing zone for the treatment of aneurysm near the bifurcation area, ${ }^{5,6)}$ (3) simultaneous EVAR and abdominal aortic replacement for the thoracic and the abdominal aortic aneurysms, ${ }^{7)}$ (4) open-stent procedure where a stent graft is inserted through the aorta for treatment of a distal aortic arch aneurysm with extending to the descending aorta.

According to the above classification, of 15 patients in our series, type (1) hybrid procedure was employed in 5 patients, and type (3) was performed in 7 patients. In the other 3 patients, surgical replacement of thoracoabdominal aorta was undertaken as the initial procedure followed by EVAR in one patients and EVAR was performed initially followed by surgical replacement in 2 patients. All 8 patients except one with accompanying abdominal aortic aneurysm, simultaneous procedures of abdominal aortic aneurysmectomy followed by EVAR were performed. The overall in-hospital mortality rate of 198 patients who received elective surgical treatment for thoracic aortic aneurysms at our institution was $5.1 \%$, and among them, mortality rate of patients receiving total aortic arch replacement surgery was 5.8\%. On the other 
hand, the rate of major complications such as cardiovascular events and spinal cord ischemia in 577 cases treated by EVAR for thoracic and thoracoabdominal aortic aneurysmal disease was 6.4\%. In our present series of 15 patients receiving hybrid treatment for multiple aortic aneurysms, one patient died of sepsis due to methicillin resistant Staphylococcus auerus infection. The other 14 patients were discharged and carefully followed. The followup period ranged from 3 to 91 months (mean 46 months), during which time no complications have occurred. Although the follow-up periods may not be sufficient to make a conclusion, we believe that the hybrid approach to multiple aortic aneurysms patients will reduce the overall risk of surgery, while yielding satisfactory long-term results.

\section{Conclusion}

The hybrid procedure combined with conventional surgical approach and EVAR, implemented simultaneously or sequentially, is effective for the treatment of multiple aortic aneurysms and is likely to reduce or minimize the risk of simultaneous or sequential multiple surgical procedures. The in-hospital mortality as well as the follow-up results in our series were acceptable.

\section{ACKNOWLEDGEMEnTS}

The authors are indebted to Professor J. Patrick Barron of the International Medical Communications Center of Tokyo Medical University for his review of this manuscript.

\section{REFERENCES}

1) Kawaguchi S, Ishimaru S, Shimazaki T, Yokoi Y. Clinical outcomes from patients treated with endovascular stent graft for thoracic aortic aneurysms. J Am Coll Cardiol. 2004; 43: 471A.

2) Hosokawa H, Iwase T, Sato M, Yoshida Y, Ueno K, Tamaki S, et al. Successful endovasucular repair of juxtarenal and suprarenal aortic aneurysms with a branched stent graft. J Vasc Surg. 2001; 33: 1087-92.

3) Verhoeven EL, Zeebregts CJ, Kapma MR, Tielliu IF, Prins TR, van den Dungen JJ. Fenestrated and branched endovascular techniques for thoraco-abdominal aneurysm repair. J Cardiovasc Surg. 2005; 46: 131-40.

4) Yano H, Ishimaru S, Kawaguchi S, Obitsu Y. Endovascular stent grafting of the descending thoracic aorta after arch repair in acute type A dissection. Ann Thorac Surg. 2002; 73: 288-91.

5) Watanabe Y, Ishimaru S, Kawaguchi S, Shimazaki T, Yokoi Y, Ito M, et al. Successful endografting with simultaneous visceral artery bypass grafting for severely calcified thoracoabdominal aortic aneurysm. J Vasc Surg. 2002; 35: 397-399.

6) Saiki N, Ishimaru S, Kawaguchi S, Shimazaki T, Yokoi Y, Obitsu Y. Endografting facilitated by axillary-axillary bypass for distal arch aneurysm after left internal thoracic artery to left anterior descending artery bypass surgery. J Thorac Cardiovasc Surg. 2003; 125: 950-2.

7) Moon MR, Mitchell RS, Dake MD, Zarins CK, Fann JI, Miller DC. Simultaneous abdominal aortic replacement and thoracic stent-graft placement for multilevel aortic disease. J Vasc Surg. 1997; 25: 332-40. 